\title{
Stability of Exponential Functional Equations with Involutions
}

\author{
Jaeyoung Chung' and Soon-Yeong Chung ${ }^{2}$ \\ ${ }^{1}$ Department of Mathematics, Kunsan National University, Kunsan 573-701, Republic of Korea \\ ${ }^{2}$ Department of Mathematics, Sogang University, Seoul 121-742, Republic of Korea
}

Correspondence should be addressed to Soon-Yeong Chung; sychung@sogang.ac.kr

Received 29 January 2014; Accepted 18 April 2014; Published 30 April 2014

Academic Editor: Ajda Fošner

Copyright (C) 2014 J. Chung and S.-Y. Chung. This is an open access article distributed under the Creative Commons Attribution License, which permits unrestricted use, distribution, and reproduction in any medium, provided the original work is properly cited.

Let $S$ be a commutative semigroup if not otherwise specified and $f: S \rightarrow \mathbb{R}$. In this paper we consider the stability of exponential functional equations $|f(x+\sigma(y))-g(x) f(y)| \leq \phi(x)$ or $\phi(y),|f(x+\sigma(y))-f(x) g(y)| \leq \phi(x)$ or $\phi(y)$ for all $x, y \in S$ and where $\sigma: S \rightarrow S$ is an involution. As main results we investigate bounded and unbounded functions satisfying the above inequalities. As consequences of our results we obtain the Ulam-Hyers stability of functional equations (Chung and Chang (in press); Chávez and Sahoo (2011); Houston and Sahoo (2008); Jung and Bae (2003)) and a generalized result of Albert and Baker (1982).

\section{Introduction}

Throughout this paper we denote by $\mathbb{R}, \mathbb{R}^{+}, \mathbb{C}, \mathbb{R}^{n}$ the set of real numbers, nonnegative real numbers, complex numbers, and the $n$-dimensional Euclidean space, respectively, and $\phi$ : $S \rightarrow \mathbb{R}^{+}, \epsilon \geq 0$. A function $m: S \rightarrow \mathbb{C}$ is called exponential provided that $m(x+y)=m(x) m(y)$ for all $x, y \in S$ and $\sigma: S \rightarrow S$ is called an involution provided that $\sigma(x+y)=$ $\sigma(x)+\sigma(y)$ and $\sigma(\sigma(x))=x$ for all $x, y \in S$. In [1], Baker proved the stability of the exponential functional equation: let $f: S \rightarrow \mathbb{C}$ satisfy the exponential functional inequality

$$
|f(x+y)-f(x) f(y)| \leq \epsilon
$$

for all $x, y \in S$. Then, either $f$ is a bounded function satisfying

$$
|f(x)| \leq \frac{1+\sqrt{1+4 \epsilon}}{2}
$$

for all $x \in S$, or $f$ is an unbounded exponential function (see also Baker et al. [2]). In particular, if $S=V$, where $V$ is a vector space over the field $\mathbb{Q}$ of rational numbers and $f: V \rightarrow \mathbb{R}$ is a bounded function satisfying (1) for $0 \leq \epsilon<1 / 4$, then $f$ satisfies either

$$
\frac{1+\sqrt{1-4 \epsilon}}{2} \leq f(x) \leq \frac{1+\sqrt{1+4 \epsilon}}{2}
$$

for all $x \in V$, or else

$$
-\epsilon \leq f(x) \leq \frac{1-\sqrt{1-4 \epsilon}}{2}
$$

for all $x \in V$ (see Albert and Baker [3]). In [4-6], some functional equations arising from number theory or product of matrices are introduced. The equations therein can be reduced to the functional equations with involution $\sigma: S \rightarrow$ $S$ :

$$
f(x+\sigma(y))-f(x) f(y)=0
$$

for all $x, y \in S$ (see Section 4). As we see in Section 4, the equations in [4-6] can be reduced to the equation of the form (5) when $S=\mathbb{C}$ with operation of multiplication and $\sigma(z)=\bar{z}$ for all $z \in \mathbb{C}$ or $\sigma(z)=z$ for all $z \in \mathbb{C}$ and the equations in [6] are reduced to those forms (5) in the complex numbers with $\sigma(z)=\bar{z}$ and in the set of quaternions with $\sigma(q)=q^{*}$, where $q^{*}$ denotes the conjugate of the quaternion $q$.

As main results of the paper, we consider, in Section 2, the Ulam-Hyers stability of exponential functional equations with involution $\sigma: S \rightarrow S$ :

$$
\begin{aligned}
& |f(x+\sigma(y))-g(x) f(y)| \leq \phi(x), \\
& |f(x+\sigma(y))-f(x) g(y)| \leq \phi(y),
\end{aligned}
$$




$$
\begin{gathered}
|f(x+\sigma(y))-g(x) f(y)| \leq \phi(y), \\
|f(x+\sigma(y))-f(x) g(y)| \leq \phi(x)
\end{gathered}
$$

for all $x, y \in S$. In Section 3, as a consequence of our main results we obtain the behaviors of bounded functions $f$ satisfying each of the inequalities

$$
\begin{gathered}
|f(x+\sigma(y))-f(x) f(y)| \leq \phi(x), \\
|f(x+\sigma(y))-f(x) f(y)| \leq \phi(y)
\end{gathered}
$$

for all $x, y \in S$. Also, as a direct consequence of the stability of (7) we obtain a generalized version of the result of Albert and Baker [3]. Our method of proof also works for investigation of bounded solutions of some other functional inequalities (see [7-9]). We refer to [7-12] for the exponential functional equations, inequalities, and related results.

In Section 4, as an application of our result, we obtain the stability of the functional equations:

$$
f\left(x_{1}, y_{1}\right) f\left(x_{2}, y_{2}\right)=f\left(x_{1} x_{2}+y_{1} y_{2}, x_{1} y_{2}-x_{2} y_{1}\right)
$$

for all $x_{1}, y_{1}, x_{2}, y_{2} \in \mathbb{R}$, and

$$
\begin{array}{r}
f\left(x_{1}, y_{1}, u_{1}, v_{1}\right) f\left(x_{2}, y_{2}, u_{2}, v_{2}\right) \\
=f\left(x_{1} x_{2}+y_{1} y_{2}+u_{1} u_{2}+v_{1} v_{2},\right. \\
x_{1} y_{2}-y_{1} x_{2}+u_{1} v_{2}-v_{1} u_{2}, \\
x_{1} u_{2}-y_{1} v_{2}-u_{1} x_{2}+v_{1} y_{2}, \\
\left.x_{1} v_{2}+y_{1} u_{2}-u_{1} y_{2}-v_{1} x_{2}\right)
\end{array}
$$

for all $x_{1}, y_{1}, u_{1}, v_{1}, x_{2}, y_{2}, u_{2}, v_{2} \in \mathbb{R}$, where $f: \mathbb{R}^{2} \rightarrow \mathbb{R}$ and $g: \mathbb{R}^{4} \rightarrow \mathbb{R}$. As stated in [6], (8) and (9) arise from a well-known theorem in number theory.

Reducing (8) and (9) to those in the complex numbers and the quaternions, we obtain the stability of (8) and (9); that is, we investigate bounded and unbounded functions $f$ satisfying each of the following functional inequalities:

$$
\begin{aligned}
& \left|f\left(x_{1}, y_{1}\right) f\left(x_{2}, y_{2}\right)-f\left(x_{1} x_{2}+y_{1} y_{2}, x_{1} y_{2}-x_{2} y_{1}\right)\right| \\
& \quad \leq \phi\left(x_{2}, y_{2}\right), \\
& \left|f\left(x_{1}, y_{1}\right) f\left(x_{2}, y_{2}\right)-f\left(x_{1} x_{2}+y_{1} y_{2}, x_{1} y_{2}-x_{2} y_{1}\right)\right| \\
& \quad \leq \phi\left(x_{1}, y_{1}\right)
\end{aligned}
$$

for all $x_{1}, y_{1}, x_{2}, y_{2} \in \mathbb{R}$ and for some $\phi: \mathbb{R}^{2} \rightarrow \mathbb{R}^{+}$, and we investigate bounded and unbounded functions $g$ satisfying each of the following functional inequalities:

$$
\begin{array}{r}
\mid g\left(x_{1}, y_{1}, u_{1}, v_{1}\right) g\left(x_{2}, y_{2}, u_{2}, v_{2}\right) \\
-g\left(x_{1} x_{2}+y_{1} y_{2}+u_{1} u_{2}+v_{1} v_{2},\right. \\
x_{1} y_{2}-y_{1} x_{2}+u_{1} v_{2}-v_{1} u_{2}, \\
x_{1} u_{2}-y_{1} v_{2}-u_{1} x_{2}+v_{1} y_{2}, \\
\left.x_{1} v_{2}+y_{1} u_{2}-u_{1} y_{2}-v_{1} x_{2}\right) \mid \\
\leq \psi\left(x_{2}, y_{2}, u_{2}, v_{2}\right),
\end{array}
$$

$$
\begin{gathered}
\mid g\left(x_{1}, y_{1}, u_{1}, v_{1}\right) g\left(x_{2}, y_{2}, u_{2}, v_{2}\right) \\
-g\left(x_{1} x_{2}+y_{1} y_{2}+u_{1} u_{2}+v_{1} v_{2},\right. \\
x_{1} y_{2}-y_{1} x_{2}+u_{1} v_{2}-v_{1} u_{2}, \\
x_{1} u_{2}-y_{1} v_{2}-u_{1} x_{2}+v_{1} y_{2}, \\
\left.x_{1} v_{2}+y_{1} u_{2}-u_{1} y_{2}-v_{1} x_{2}\right) \mid \\
\leq \psi\left(x_{1}, y_{1}, u_{1}, v_{1}\right)
\end{gathered}
$$

for all $x_{1}, y_{1}, u_{1}, v_{1}, x_{2}, y_{2}, u_{2}, v_{2} \in \mathbb{R}$ and for some $\psi: \mathbb{R}^{4} \rightarrow$ $\mathbb{R}^{+}$. We also refer the reader to [5] for another proof of finding general solutions of (8) and refer to [13] for the stability of inequalities (10) (13).

\section{Stability of (6)}

Throughout this section we assume that $S$ is a commutative semigroup if not otherwise specified. An exponential function $m: S \rightarrow \mathbb{C}$ is called $\sigma$-exponential if $m$ satisfies $m(\sigma(x))=m(x)$ for all $x \in S$. We denote $\sigma$-exponential functions $m$ by $m_{\sigma}$. It is easy to see that if $S$ is uniquely 2divisible (i.e., for each $x \in S$ there exists a unique $y \in S$ such that $2 y=x$; we write $y=x / 2$ ), then $m_{\sigma}$ is a $\sigma$-exponential function if and only if

$$
m_{\sigma}(x)=m\left(\frac{x+\sigma(x)}{2}\right), \quad x \in S
$$

for some exponential function $m: S \rightarrow \mathbb{C}$. Throughout this section we denote by $\sigma: S \rightarrow S$ an involution. In the following we exclude the trivial cases when $f=0$ or $g=0$.

Theorem 1. Let $f, g: S \rightarrow \mathbb{C}$ satisfy

$$
|f(x+\sigma(y))-g(x) f(y)| \leq \phi(x)
$$

for all $x, y \in S$. If $S$ is a group and $f$ is bounded, then

$$
|1-| g(x)|| \leq \frac{\phi(x)}{M_{f}}
$$

for all $x \in S$, where $M_{f}=\sup _{x \in S}|f(x)|$. If $f$ is unbounded, then there exists a $\sigma$-exponential function $m_{\sigma}: S \rightarrow \mathbb{C}$ such that

$$
\begin{gathered}
g(x)=g(0) m_{\sigma}(x), \\
\left|f(x)-f(0) g(0) m_{\sigma}(x)\right| \leq \phi(x)
\end{gathered}
$$

for all $x \in S$, where $g(0)= \pm 1$. In particular, if $\phi(x)=\epsilon \geq 0$ for all $x \in S$, then we have

$$
g(x)=m_{\sigma}(x), \quad f(x)=f(0) m_{\sigma}(x)
$$

for all $x \in S$.

Proof. First, we assume that $f$ is bounded (in this case we assume that $S$ is a group). Using the triangle inequality with (15) we have

$$
|g(x) f(y)| \leq|f(x+\sigma(y))|+\phi(x) \leq M_{f}+\phi(x)
$$


for all $x, y \in S$. Taking the supremum of the left-hand side of (19) with respect to $y$ we have

$$
|g(x)| M_{f} \leq M_{f}+\phi(x)
$$

for all $x \in S$, which implies

$$
M_{f}(|g(x)|-1) \leq \phi(x)
$$

for all $x \in S$. Replacing $y$ by $\sigma(-x+y)$ in (15) and using the triangle inequality with the result we have

$$
|f(y)| \leq|g(x) f(\sigma(-x+y))| \leq|g(x)| M_{f}+\phi(x)
$$

for all $x, y \in S$. Taking the supremum of the left-hand side of (22) with respect to $y$ we have

$$
M_{f} \leq|g(x)| M_{f}+\phi(x)
$$

for all $x \in S$, which implies

$$
M_{f}(1-|g(x)|) \leq \phi(x)
$$

for all $x \in S$. From (21) and (24) we get (16). Now, we assume that $f$ is unbounded. Putting $x=0$ in (15) we have

$$
|f(\sigma(y))-g(0) f(y)| \leq \phi(0)
$$

for all $y \in S$. Now, using the triangle inequality, (15), and (25) we have

$$
\begin{aligned}
& |f(z)||g(y) g(x)-g(0) g(x+\sigma(y))| \\
& \leq|g(y) g(x) f(z)-g(y) f(x+\sigma(z))| \\
& \quad+|g(y) f(x+\sigma(z))-f(y+\sigma(x)+z)| \\
& \quad+|f(y+\sigma(x)+z)-g(0) f(x+\sigma(y)+\sigma(z))| \\
& \quad+|g(0) f(x+\sigma(y)+\sigma(z))-g(0) g(x+\sigma(y)) f(z)| \\
& \leq|g(y)| \phi(x)+\phi(y)+\phi(0)+|g(0)| \phi(x+\sigma(y))
\end{aligned}
$$

for all $x, y, z \in S$. Since $f$ is unbounded, it follows from (26) that

$$
g(x) g(y)=g(0) g(x+\sigma(y))
$$

for all $x, y \in S$. Dividing (27) by $g(0)^{2}$ we have

$$
g_{0}(x) g_{0}(y)=g_{0}(x+\sigma(y))
$$

for all $x, y \in S$, where $g_{0}(x)=g(x) / g(0)$. Thus, $g_{0}$ is $\sigma$ exponential and $g(x)=g(0) m_{\sigma}(x)$ for some $\sigma$-exponential $m_{\sigma}$. Replacing $y$ by $\sigma(y)$ in (25) and multiplying $g(0)$ in the result we have

$$
\left|g(0) f(y)-g(0)^{2} f(\sigma(y))\right| \leq|g(0)| \phi(0)
$$

for all $y \in S$. Using the triangle inequality with (25) and (29) we have

$$
\left|1-g(0)^{2}\right||f(\sigma(y))| \leq(1+|g(0)|) \phi(0)
$$

for all $y \in S$. Since $f$ is unbounded, we have $g(0)^{2}=1$. Putting $y=0$ in (15) we have

$$
|f(x)-f(0) g(x)| \leq \phi(x)
$$

for all $x \in S$. Thus, we get (17). For the particular case when $\phi(x)=\epsilon$ for all $x \in S$, replacing $(x, y)$ by $(\sigma(x), y)$ and $(x, y)$ by $(\sigma(y), x)$ in (15) and using triangle inequality with the resulting two inequalities, we have

$$
|f(x) g(\sigma(y))-f(y) g(\sigma(x))| \leq 2 \epsilon
$$

for all $x, y \in S$, which is

$$
|f(x) g(y)-f(y) g(x)| \leq 2 \epsilon
$$

for all $x, y \in S$, since $g(x)=g(\sigma(x))$ for all $x \in S$. Dividing both sides of (33) by $|g(y)|$ we have

$$
|f(x)-\alpha(y) g(x)| \leq \frac{2 \epsilon}{|g(y)|}
$$

for all $y \in J:=\{y: g(y) \neq 0\}$, where $\alpha(y)=f(y) / g(y)$. Since $f$ is unbounded, we have $\alpha(y) \neq 0$ for all $y \in J$ and hence $g$ is unbounded. Replacing $y$ by $y_{1} \in J$ and $y$ by $y_{2} \in J$ in (34) we have two inequalities. Using the triangle inequality with these two resulting inequalities we have

$$
\left|\alpha\left(y_{1}\right)-\alpha\left(y_{2}\right)\right||g(x)| \leq 2 \epsilon\left(\frac{1}{\left|g\left(y_{1}\right)\right|}+\frac{1}{\left|g\left(y_{2}\right)\right|}\right)
$$

for all $x \in S$. Since $g$ is unbounded, from (35) we have $\alpha\left(y_{1}\right)=$ $\alpha\left(y_{2}\right)$ and hence $\alpha(y):=\alpha$ is independent of $y \in J$. From (34) we have

$$
|f(x)-\alpha g(x)| \leq \frac{2 \epsilon}{|g(y)|}
$$

for all $x \in S$ and $y \in J$. Since $g$ is unbounded we have

$$
f(x)=\alpha g(x)=\alpha g(0) m_{\sigma}(x)
$$

for all $x \in S$. Putting (37) in (15) we have

$$
|\alpha g(0)(1-g(0))|\left|m_{\sigma}(x) m_{\sigma}(y)\right| \leq \epsilon
$$

for all $x, y \in S$. Since $\alpha \neq 0, g(0) \neq 0$, and $m_{\sigma}$ is unbounded, we have $g(0)=1$. Thus, we get (18). This completes the proof.

Remark 2. We have no idea if the case $g(x)=-m_{\sigma}(x)$ in (17) occurs or not. For example, it can be verified that if there exists a sequence $x_{n} \in S, n=1,2,3, \ldots$, such that $\left(1+\phi\left(x_{n}\right)\right) /\left|g\left(x_{n}\right)\right| \rightarrow 0$ as $n \rightarrow \infty$, the case $g(x)=-m_{\sigma}(x)$ in (17) does not occur; in particular $(g, f)$ satisfies (18).

Remark 3. In general, the inequality (16) cannot be replaced by the weaker inequality:

$$
|1-g(x)| \leq \frac{\epsilon}{M_{f}}
$$


for all $x \in S$. Indeed, let $\phi(x)=\epsilon>0, \sigma(y)=y, f(x)=$ $\epsilon \sin x, g(x)=\cos x$ for all $x, y \in S$. Then we have

$$
|f(x+y)-f(x) g(y)|=|\epsilon \cos x \sin y| \leq \epsilon
$$

for all $x, y \in S$. However, the inequality (39) fails since

$$
|1-g(\pi)|=|1-\cos \pi|=2>1=\frac{\epsilon}{M_{f}} .
$$

As a direct consequence of Theorem 1 we have the following.

Corollary 4. Let $f: S \rightarrow \mathbb{C}$ satisfy

$$
|f(x+\sigma(y))-f(x) f(y)| \leq \phi(x)
$$

for all $x, y \in S$. If $S$ is a group and $f$ is bounded, then

$$
|f(y)||1-| f(x)|| \leq \phi(x)
$$

for all $x, y \in S$. If $f$ is unbounded, then $f$ is $\sigma$-exponential.

Proof. The inequality (16) implies (43). Now, by Theorem 1, we have

$$
f(x)=f(0) m_{\sigma}(x), \quad x \in S
$$

for some $\sigma$-exponential function $m_{\sigma}$. Putting (44) to (42) and letting $x=0$ we have

$$
|f(0)(1-f(0))|\left|m_{\sigma}(y)\right| \leq \phi(0)
$$

for all $y \in S$. Since $f$ is unbounded, from (45) we have $f(0)=$ 1. This completes the proof.

Theorem 5. Let $f, g: S \rightarrow \mathbb{C}$ satisfy

$$
|f(x+\sigma(y))-f(x) g(y)| \leq \phi(y)
$$

for all $x, y \in S$. If $S$ is a group and $f$ is bounded, then

$$
|1-| g(x)|| \leq \frac{\phi(x)}{M_{f}}
$$

for all $x \in S$, where $M_{f}=\sup _{x \in S}|f(x)|$. If $f$ is unbounded, then there exists an exponential function $m: S \rightarrow \mathbb{C}$ such that

$$
g(x)=m(x), \quad|f(x)-f(0) m(\sigma(x))| \leq \phi(\sigma(x))
$$

for all $x \in S$. In particular, if $\phi(x)=\epsilon \geq 0$ for all $x \in S$, then we have

$$
g(x)=m(x), \quad f(x)=f(0) m(\sigma(x))
$$

for all $x \in S$.

Proof. Using the same method as in the proof of Theorem 1, we can show that if $f$ is bounded and $S$ is a group, then $f$ satisfies (47). Assume that $f$ is unbounded. Using the triangle inequality and (46) we have

$$
\begin{aligned}
& |f(z)||g(x) g(y)-g(y+x)| \\
& \leq|f(z) g(x) g(y)-f(z+\sigma(x)) g(y)| \\
& \quad+|f(z+\sigma(x)) g(y)-f(z+\sigma(x)+\sigma(y))| \\
& \quad+|f(z+\sigma(y+x))-f(z) g(y+x)| \\
& \leq \phi(x)|g(y)|+\phi(y)+\phi(x+y)
\end{aligned}
$$

for all $x, y, z \in S$. Since $f$ is unbounded, it follows from (50) that

$$
g(x+y)=g(x) g(y)
$$

for all $x, y \in S$. Therefore, $g$ is an exponential function: say $g=m$. Putting $x=0$ and replacing $y$ by $\sigma(x)$ in (46) we get (48). Assume that $\phi(y)=\epsilon$ for all $y \in S$. Replacing $(x, y)$ by $(x, \sigma(y))$ and $(x, y)$ by $(y, \sigma(x))$ in (46) using triangle inequality with the resulting two inequalities we have

$$
|f(x) g(\sigma(y))-f(y) g(\sigma(x))| \leq 2 \epsilon
$$

for all $x, y \in S$. Now, using the same method as in the proof of Theorem 1 (after the inequality (33)) we can show that

$$
f(x)=\alpha g(\sigma(x))
$$

for all $x \in S$. Since $g$ is an exponential function, we have $g(\sigma(0))=g(0)=1$ and hence $\alpha=f(0)$. This completes the proof.

As a direct consequence of Theorem 5 we have the following.

Corollary 6. Let $f: S \rightarrow \mathbb{C}$ satisfy

$$
|f(x+\sigma(y))-f(x) f(y)| \leq \phi(y)
$$

for all $x, y \in S$. If $S$ is a group and $f$ is bounded, then

$$
|f(y)||1-| f(x)|| \leq \phi(x)
$$

for all $x, y \in S$. If $f$ is unbounded, then $f$ is $\sigma$-exponential.

Proof. If $f$ is bounded, the inequality (55) follows from (47). If $f$ is unbounded, then, by Theorem $5, f$ is exponential. Thus, from (54) we have

$$
|f(x)||f(\sigma(y))-f(y)| \leq \phi(y)
$$

for all $x, y \in S$. Since $f$ is unbounded, it follows from (56) that $f(y)=f(\sigma(y))$ for all $y \in S$. This completes the proof.

Theorem 7. Let $f, g: S \rightarrow \mathbb{C}$ satisfy

$$
|f(x+\sigma(y))-g(x) f(y)| \leq \phi(y)
$$

for all $x, y \in S$. If $f(0)=0$, then

$$
|f(x)| \leq \phi(0), \quad|g(x)| \leq \inf _{y \in S} \frac{\phi(y)+\phi(0)}{|f(y)|}
$$


for all $x \in S$. If $f(0) \neq 0, S$ is a group, and $g$ is bounded, then

$$
|| f(0)|-| f(x)|| \leq \frac{\phi(x)+\phi(0)}{M_{g}}
$$

for all $x \in S$, where $M_{g}=\sup _{x \in S}|g(x)|$. If $f(0) \neq 0$ and $g$ is unbounded, then there exists an unbounded $\sigma$-exponential function $m_{\sigma}: S \rightarrow \mathbb{C}$ such that

$$
f(x)=f(0) m_{\sigma}(x), \quad\left|g(x)-m_{\sigma}(x)\right| \leq \frac{\phi(0)}{|f(0)|}
$$

for all $x \in S$.

Proof. Putting $y=0$ in (57) we have

$$
|f(x)-f(0) g(x)| \leq \phi(0)
$$

for all $x \in S$. If $f(0)=0$, then we have

$$
|f(x)| \leq \phi(0)
$$

for all $x \in S$. Using the triangle inequality with (57) and using (62) we have

$$
|g(x) f(y)| \leq \phi(y)+\phi(0)
$$

for all $x, y \in S$. Dividing (63) by $|f(y)|$ and taking infimum of the right-hand side of the result we get

$$
|g(x)| \leq \inf _{y \in S} \frac{\phi(y)+\phi(0)}{|f(y)|}
$$

for all $x \in S$, which gives (58). If $f(0) \neq 0$, using the triangle inequality with (57), using (61), and dividing the result by $|f(0)|$, we have

$$
\left|g(x+\sigma(y))-g(x) f_{0}(y)\right| \leq \phi_{0}(y)
$$

for all $x, y \in S$, where $f_{0}(y)=f(y) / f(0), \phi_{0}(y)=$ $(\phi(y)+\phi(0)) /|f(0)|$. If $g$ is bounded and $S$ is a group, then by Theorem $5, f_{0}$ satisfies

$$
|1-| f_{0}(x)|| \leq \frac{\phi_{0}(x)}{M_{g}}
$$

for all $x \in S$, where $M_{g}=\sup _{x \in S}|g(x)|$. Multiplying (66) by $|f(0)|$ we have

$$
\|f(0)|-| f(x)\| \leq \frac{\phi(x)+\phi(0)}{M_{g}}
$$

for all $x \in S$. If $g$ is unbounded, then by Theorem $5, f_{0}$ is exponential function; say $f_{0}=m$. Putting $y=0$ in (57) and dividing the result by $|f(0)|$ we have

$$
|g(x)-m(x)| \leq \frac{\phi(0)}{|f(0)|}
$$

for all $x \in S$. Multiplying (68) by $|f(y)|$,

$$
|g(x) f(y)-m(x) f(y)| \leq \frac{\phi(0)|f(y)|}{|f(0)|}
$$

for all $x, y \in S$. Using the triangle inequality with (57) and (69) we have

$$
\begin{aligned}
\mid f(0) & m(x)|| m(\sigma(y))-m(y) \mid \\
= & |f(x+\sigma(y))-m(x) f(y)| \\
\leq & |f(x+\sigma(y))-g(x) f(y)| \\
& +|g(x) f(y)-m(x) f(y)| \\
\leq & \phi(y)+\frac{\phi(0)|f(y)|}{|f(0)|}
\end{aligned}
$$

for all $x, y \in S$. Since $m$ is unbounded, it follows from (70) that $m(\sigma(y))=m(y)$ for all $y \in S$. This completes the proof.

Theorem 8. Let $f, g: S \rightarrow \mathbb{C}$ satisfy

$$
|f(x+\sigma(y))-f(x) g(y)| \leq \phi(x)
$$

for all $x, y \in S$. If $f(0)=0$, then

$$
|f(x)| \leq \phi(0), \quad|g(x)| \leq \inf _{y \in S} \frac{\phi(y)+\phi(0)}{|f(y)|}
$$

for all $x \in S$. If $f(0) \neq 0, S$ is a group, and $g$ is bounded, then

$$
\|f(0)|-| f(x)\| \leq \frac{\phi(x)+\phi(0)}{M_{g}}
$$

for all $x \in S$, where $M_{g}=\sup _{x \in S}|g(x)|$. If $f(0) \neq 0$ and $g$ is unbounded, then there exists an unbounded exponential $m$ : $S \rightarrow \mathbb{C}$ such that

$$
f(x)=f(0) m(x), \quad|g(x)-m(\sigma(x))| \leq \frac{\phi(0)}{|f(0)|}
$$

for all $x \in S$.

Proof. Putting $x=0$ in (71) we have

$$
|f(\sigma(y))-f(0) g(y)| \leq \phi(0)
$$

for all $y \in S$. If $f(0)=0$, then replacing $y$ by $\sigma(y)$ in (75) we have

$$
|f(y)| \leq \phi(0)
$$

for all $y \in S$. Using the triangle inequality with (71) and using (76) we have

$$
|f(x) g(y)| \leq \phi(x)+\phi(0)
$$

for all $x, y \in S$. Dividing (77) by $|f(x)|$ and taking infimum of the right-hand side of the result we have

$$
|g(y)| \leq \inf _{x \in S} \frac{\phi(x)+\phi(0)}{|f(x)|}
$$


for all $x \in S$, which gives (72). If $f(0) \neq 0$, using the triangle inequality with (71) and (75), replacing $(x, y)$ by $(y, x)$, and dividing the result by $|f(0)|$, we have

$$
\left|g(x+\sigma(y))-g(x) f_{0}(y)\right| \leq \phi_{0}(y)
$$

for all $x, y \in S$, where $f_{0}(y)=f(y) / f(0), \phi_{0}(y)=$ $(\phi(y)+\phi(0)) /|f(0)|$. If $g$ is bounded and $S$ is a group, then by Theorem 5 we get (73). If $g$ is unbounded, then by Theorem 5 , $f_{0}$ is exponential; say $f_{0}=m$. Putting $x=0$ in (71), replacing $y$ by $\sigma(x)$ in the result, and dividing the result by $|f(0)|$ we get (74). This completes the proof.

Finally, we consider the functional inequality with three unknown functions $f, g, h: S \rightarrow \mathbb{C}$ :

$$
|f(x+\sigma(y))-g(x) h(y)| \leq \epsilon
$$

for all $x, y \in S$. The inequality (80) is no more than the inequality

$$
|f(x+y)-g(x) h(y)| \leq \epsilon
$$

for all $x, y \in S$, since, if we replace $y$ by $\sigma(y)$ in (80) and $h$ by $h \circ \sigma$, inequality (80) is reduced to (81).

In the following we exclude the trivial cases when $g=0$ or $h=0$.

Theorem 9. Let $f, g, h: S \rightarrow \mathbb{C}$ satisfy the inequality(81). If $g$ or $h$ is unbounded, then there exists an unbounded exponential function $m: S \rightarrow \mathbb{C}$ such that

$$
\begin{gathered}
g(x)=g(0) m(x), \quad h(x)=h(0) m(x), \\
|f(x)-g(0) h(0) m(x)| \leq \epsilon
\end{gathered}
$$

for all $x \in S$. If $S$ is a group and $g$ or $h$ is bounded, then

$$
\begin{gathered}
|| h(x)|-| h(0)\left\|\leq \frac{2 \epsilon}{M_{g}}, \quad\right\| g(x)|-| g(0) \| \leq \frac{2 \epsilon}{M_{h}}, \\
\|f(x)|-| g(0) h(0)\| \leq 3 \epsilon
\end{gathered}
$$

for all $x \in S$, where $M_{g}=\sup _{x \in S}|g(x)|, M_{h}=\sup _{x \in S}|h(x)|$.

Proof. Replacing $(x, y)$ by $(y, x)$ in $(81)$ and using the triangle inequality with the result and (81) we have

$$
|g(x) h(y)-h(x) g(y)| \leq 2 \epsilon
$$

for all $x, y \in S$. Since we exclude the trivial cases when $g=0$ or $h=0$, it follows from (84) that $g$ is bounded if and only if $h$ is bounded. If $h(0)=0$, putting $y=0$ in (81), and if $h(0)=0$, putting $y=0$ in (81), we have $|f(x)| \leq \epsilon$ for all $x \in S$. Using the triangle inequality with (81) we have

$$
|g(x) h(y)| \leq|f(x+y)|+\epsilon \leq 2 \epsilon
$$

for all $x, y \in S$. Thus, we have

$$
M_{g} M_{h} \leq 2 \epsilon
$$

Assume that $g$ or $h$ is unbounded. From $(86)$ we have $g(0) \neq 0$, $h(0) \neq 0$. Putting $y=0$ and replacing $x$ by $x+y$ in (81) we have

$$
|f(x+y)-g(x+y) h(0)| \leq \epsilon
$$

for all $x, y \in S$. Using the triangle inequality with (81) and (87) and dividing the result by $|h(0)|$ we have

$$
\left|g(x+y)-g(x) h_{0}(y)\right| \leq \frac{2 \epsilon}{|h(0)|}
$$

for all $x, y \in S$, where $h_{0}(x)=h(x) / h(0)$. Replacing $y$ by $\sigma(y)$ in (87) and using Theorem 5 we get

$$
h_{0}(x)=m(x), \quad g(x)=g(0) m(x), \quad \forall x \in S
$$

for some exponential function $m$. Putting (89) in (81) we obtain (82). Now, we assume that $g$ or $h$ is bounded. If $h(0) \neq 0$, then using Theorem 5 with (88) we have

$$
|| h(x)|-| h(0) \| \leq \frac{2 \epsilon}{M_{g}}
$$

for all $x \in S$. If $g(0)=0$, from (86) we have

$$
|g(x)| \leq \frac{2 \epsilon}{M_{h}}
$$

for all $x \in S$. Thus, $h$ satisfies (90) for both cases $h(0)=0$ and $h(0) \neq 0$. Changing the role of $g$ and $h$ we get

$$
\|g(x)|-| g(0)\| \leq \frac{2 \epsilon}{M_{h}}
$$

for all $x \in S$. Putting $y=0$ in (81) and using the triangle inequality we have

$$
\|f(x)|-| h(0) g(x)\| \leq \epsilon
$$

for all $x \in S$. Multiplying $|h(0)|$ in (92) we have

$$
|| h(0) g(x)|-| g(0) h(0) \| \leq \frac{2 \epsilon|h(0)|}{M_{h}} \leq 2 \epsilon
$$

for all $x \in S$. From (92) and (93), using the triangle inequality we have

$$
|| f(x)|-| g(0) h(0) \| \leq 3 \epsilon
$$

for all $x \in S$. This completes the proof.

\section{Bounded Solutions}

Throughout this section, we assume that $G$ is a group and $\sigma: S \rightarrow S$ an involution. We describe bounded functions satisfying following functional inequalities:

$$
\begin{gathered}
|f(x+\sigma(y))-f(x) f(y)| \leq \phi(x), \\
|f(x+\sigma(y))-f(x) f(y)| \leq \phi(y)
\end{gathered}
$$

for all $x, y \in G$ and for some $\phi: G \rightarrow \mathbb{R}^{+}$. 
Theorem 10. Let $f: G \rightarrow \mathbb{C}$ be bounded functions satisfying (96) or (97). Then $f$ satisfies

$$
|f(x)| \leq \frac{1}{2}(1+\sqrt{1+4 \phi(x)})
$$

for all $x \in G$. Let $K:=\{x \in G: \phi(x)<1 / 4\}$. Then, either $f$ satisfies

$$
\frac{1}{2}(1+\sqrt{1-4 \phi(x)}) \leq|f(x)| \leq \frac{1}{2}(1+\sqrt{1+4 \phi(x)})
$$

for all $x \in K$, or else

$$
|f(x)| \leq \frac{1}{2}(1-\sqrt{1-4 \phi(x)})
$$

for all $x \in K$.

Proof. By Corollaries 4 and 6, every bounded solution of (96) and (97) satisfies

$$
|f(y)(1-|f(x)|)| \leq \phi(x)
$$

for all $x, y \in G$. Letting $y=x$ in (101) we have

$$
-\phi(x) \leq|f(x)|(|f(x)|-1) \leq \phi(x)
$$

for all $x \in G$. Solving the inequality (102) we get (98). Also from (102), for each $x \in K$, we have

$$
\frac{1}{2}(1+\sqrt{1-4 \phi(x)}) \leq|f(x)| \leq \frac{1}{2}(1+\sqrt{1+4 \phi(x)}),
$$

or

$$
|f(x)| \leq \frac{1}{2}(1-\sqrt{1-4 \phi(x)}) .
$$

Assume that there exist $x_{1}, x_{2} \in K$ such that

$$
\begin{aligned}
& \left|f\left(x_{1}\right)\right| \leq \frac{1}{2}\left(1-\sqrt{1-4 \phi\left(x_{1}\right)}\right), \\
& \left|f\left(x_{2}\right)\right| \geq \frac{1}{2}\left(1+\sqrt{1-4 \phi\left(x_{2}\right)}\right) .
\end{aligned}
$$

Putting $y=x_{2}, x=x_{1}$ in (101) we have the contradiction

$$
\frac{1}{4}<\left|f\left(x_{2}\right)\left(1-\left|f\left(x_{1}\right)\right|\right)\right| \leq \phi\left(x_{2}\right)<\frac{1}{4} .
$$

Thus, $f$ satisfies (103) for all $x \in K$ or (104) for all $x \in K$. This completes the proof.

Define $\sigma: \mathbb{R}^{2} \rightarrow \mathbb{R}^{2}$ by $\sigma(x, y)=(y, x)$ for all $x, y \in \mathbb{R}$. Then it is easy to see that every $\sigma$-exponential $m_{\sigma}: \mathbb{R}^{2} \rightarrow \mathbb{C}$ has the form $m_{\sigma}(x, y)=m(x+y), x, y \in \mathbb{R}$ for some exponential $m: \mathbb{R} \rightarrow \mathbb{C}$. Thus, by Corollary 4 and Theorem 10, we have the following.
Example 11. Let $f: \mathbb{R}^{2} \rightarrow \mathbb{C}$ satisfy

$$
|f(x+v, y+u)-f(x, y) f(u, v)| \leq \frac{1}{4\left(u^{2}+v^{2}\right)}
$$

for all $x, y, u, v \in \mathbb{R}$. Then, if $f$ is unbounded function, then $f$ has the form

$$
f(x, y)=m(x+y)
$$

for all $x, y \in \mathbb{R}$, where $m: \mathbb{R} \rightarrow \mathbb{C}$ is an exponential function; if $f$ is bounded, then $f$ satisfies

$$
|f(x, y)| \leq \frac{1}{2}\left(1+\sqrt{1+\frac{1}{\left(x^{2}+y^{2}\right)}}\right)
$$

for all $x, y \in \mathbb{R}$. Let $K=\left\{(x, y): x^{2}+y^{2}>1\right\}$. Then we have

$$
\begin{aligned}
& \frac{1}{2}\left(1+\sqrt{1-\frac{1}{\left(x^{2}+y^{2}\right)}}\right) \leq|f(x, y)| \\
& \leq \frac{1}{2}\left(1+\sqrt{1+\frac{1}{\left(x^{2}+y^{2}\right)}}\right)
\end{aligned}
$$

for all $x, y \in K$, or

$$
|f(x, y)| \leq \frac{1}{2}\left(1-\sqrt{1-\frac{1}{\left(x^{2}+y^{2}\right)}}\right)
$$

for all $x, y \in K$.

Corollary 12. Let $\epsilon<1 / 4$ and $f: G \rightarrow \mathbb{C}$ be a bounded function satisfying

$$
|f(x+\sigma(y))-f(x) f(y)| \leq \epsilon
$$

for all $x, y \in G$. Then either $f$ satisfies

$$
\frac{1}{2}(1+\sqrt{1-4 \epsilon}) \leq|f(x)| \leq \frac{1}{2}(1+\sqrt{1+4 \epsilon})
$$

for all $x \in G$, or else

$$
|f(x)| \leq \frac{1}{2}(1-\sqrt{1-4 \epsilon})
$$

for all $x \in G$.

In particular, if $G$ is 2-divisible and $\sigma(x)=x$ for all $x \in$ $G$, then we obtain the following result which is a generalized version of the result of Albert and Baker [3].

Corollary 13. Let $S$ be 2 -divisible, $\epsilon<1 / 4$, and let $f: G \rightarrow \mathbb{R}$ be bounded functions satisfying

$$
|f(x+y)-f(x) f(y)| \leq \epsilon
$$

for all $x, y \in G$. Then either $f$ satisfies

$$
\frac{1}{2}(1+\sqrt{1-4 \epsilon}) \leq f(x) \leq \frac{1}{2}(1+\sqrt{1+4 \epsilon})
$$

for all $x \in G$, or else

$$
-\epsilon \leq f(x) \leq \frac{1}{2}(1-\sqrt{1-4 \epsilon})
$$

for all $x \in G$. 
Proof. Replacing $y$ by $x$ in (115) and using the triangle inequality with the result we have $f(2 x) \geq f(x)^{2}-\epsilon \geq-\epsilon$ for all $x \in G$, which implies that $f(x) \geq-\epsilon$ for all $x \in G$. Since $\epsilon<(1 / 2)(1-\sqrt{1-4 \epsilon})$, inequality (113) implies (116), and (114) implies (117). This completes the proof.

\section{Applications}

In this section we consider the stability of (8) and (9) which were dealt with in [13]. Let $\mathbb{W}=\{a+b i+c j+d k \mid a, b, c, d \in \mathbb{R}\}$ be the quaternion group. Recall that $i^{2}=j^{2}=k^{2}=-1, i j=k$, $j k=i, k i=j, j i=-k, k j=-i, i k=-j$, and the conjugate of $q=a+b i+c j+d k \in \mathbb{M}$ is given by $q^{*}=a-b i-c j-d k$. We denote $\|q\|=\sqrt{q q^{*}}=\sqrt{a^{2}+b^{2}+c^{2}+d^{2}}$. We first consider unbounded functions $f: \mathbb{R}^{2} \rightarrow \mathbb{R}$ satisfying (10) and (11) and unbounded functions $g: \mathbb{R}^{4} \rightarrow \mathbb{R}$ satisfying (12) and (13).

Theorem 14. Let $f: \mathbb{R}^{2} \rightarrow \mathbb{R}$ be an unbounded function satisfying (10). Then $f$ has the form

$$
f(x, y)=m\left(\sqrt{x^{2}+y^{2}}\right)
$$

for all $x, y \in \mathbb{R}$.

Proof. Let $F(x+y i)=f(x, y)$ and $\Phi(x+y i)=\phi(x, y)$ for all $x, y \in \mathbb{R}$. Then the functional inequality (10) is converted to

$$
|F(\bar{z} w)-F(z) F(w)| \leq \Phi(w)
$$

for all $z=x_{2}+i y_{2}, w=x_{1}+i y_{1} \in \mathbb{C}$. Letting $\sigma(z)=\bar{z}$ and using Corollary 4 when $S=\langle\mathbb{C}, \cdot\rangle$ we have $F(z)=m_{\sigma}(z)$ for all $z \in \mathbb{C}$. In view of $(14), m_{\sigma}(z)$ is written in the form $m_{\sigma}(z)=$ $m(|z|)$ for all $z \in \mathbb{C}$. Thus, we get (118). This completes the proof.

Using Corollary 6 we obtain the following.

Theorem 15. Let $f: \mathbb{R}^{2} \rightarrow \mathbb{R}$ be an unbounded function satisfying (11). Then $f$ has the form

$$
f(x, y)=m\left(\sqrt{x^{2}+y^{2}}\right)
$$

for all $x, y \in \mathbb{R}$.

Theorem 16. Let $g: \mathbb{R}^{4} \rightarrow \mathbb{R}$ be an unbounded function satisfying (12). Then $g$ has the form

$$
g(x, y, u, v)=m\left(\sqrt{x^{2}+y^{2}+u^{2}+v^{2}}\right)
$$

for all $x, y, u, v \in \mathbb{R}$.

Proof. Let $F(x+y i+u j+v k)=g(x, y, u, v)$ and $\Psi(x+y i+u j+$ $v k)=\psi(x, y, u, v)$ for all $x, y, u, v \in \mathbb{R}$. Then the functional inequality (12) is reduced to

$$
\left|F(q) F(p)-F\left(q p^{*}\right)\right| \leq \Psi(q)
$$

for all $p, q \in \mathbb{H}$. Letting $\sigma(q)=q^{*}$ and using Corollary 4 when $S=\langle\mathbb{H}, \cdot\rangle$ we have that $F(q)=m_{\sigma}(q)$ for all $q \in \mathbb{M}$. In view of (14), $m_{\sigma}(q)$ is written in the form $m_{\sigma}(q)=m(\|q\|)$ for all $q \in \mathbb{H}$. Thus, we get (121). This completes the proof.

Using Corollary 6 we obtain the following.

Theorem 17. Let $g: \mathbb{R}^{4} \rightarrow \mathbb{R}$ be an unbounded function satisfying (13). Then $g$ has the form

$$
g(x, y, u, v)=m\left(\sqrt{x^{2}+y^{2}+u^{2}+v^{2}}\right)
$$

for all $x, y, u, v \in \mathbb{R}$

Using the results in Section 3 we can also obtain bounded functions $f: \mathbb{R}^{2} \rightarrow \mathbb{R}$ satisfying the inequalities (10), (11) and bounded functions $g: \mathbb{R}^{4} \rightarrow \mathbb{R}$ satisfying (12) and (13) as in [13].

\section{Conflict of Interests}

The authors declare that there is no conflict of interests regarding the publication of this paper.

\section{Acknowledgment}

This work was supported by Basic Science Research Program through the National Foundation of Korea (NRF) funded by the Korea Government (MOE): no. 2012R1A1A008507 for Jaeyoung Chung and no. 2012R1A1A2004689 for Soon-Yeong Chung.

\section{References}

[1] J. A. Baker, "The stability of the cosine equation," Proceedings of the American Mathematical Society, vol. 80, no. 3, pp. 411-416, 1980.

[2] J. Baker, J. Lawrence, and F. Zorzitto, "The stability of the equation $f(x+y)=f(x) f(y)$," Proceedings of the American Mathematical Society, vol. 74, no. 2, pp. 242-246, 1979.

[3] M. Albert and J. A. Baker, "Bounded solutions of a functional inequality," Canadian Mathematical Bulletin, vol. 25, no. 4, pp. 491-495, 1982.

[4] E. A. Chávez and P. K. Sahoo, "On a functional equation arising from number theory," Applied Mathematics Letters, vol. 24, no. 3, pp. 344-347, 2011.

[5] K. B. Houston and P. K. Sahoo, "On two functional equations and their solutions," Applied Mathematics Letters, vol. 21, no. 9, pp. 974-977, 2008.

[6] S. M. Jung and J. H. Bae, "Some functional equations originating from number theory," Proceedings of the Indian Academy of Science, vol. 113, no. 2, pp. 91-98, 2003.

[7] J. Chudziak and J. Tabor, "On the stability of the GołabSchinzel functional equation," Journal of Mathematical Analysis and Applications, vol. 302, no. 1, pp. 196-200, 2005.

[8] G. H. Kim and P. K. Sahoo, "Stability of some functional equations related to distance measures-I," Applied Mathematics Letters, vol. 24, no. 6, pp. 843-849, 2011. 
[9] J. A. Najdecki, "On stability of a functional equation connected with the Reynolds operator," Journal of Inequalities and Applications, vol. 2007, Article ID 79816, 3 pages, 2007.

[10] J. Aczél and J. Dhombres, Functional Equations in Several Variables, vol. 31, Cambridge University Press, New York, NY, USA, 1989.

[11] D. H. Hyers, G. Isac, and T. M. Rassias, Stability of Functional Equations in Several Variables, Birkhäuser, Boston, MASS, USA, 1998.

[12] P. Sinopoulos, "Functional equations on semigroups," Aequationes Mathematicae, vol. 59, no. 3, pp. 255-261, 2000.

[13] J. Chung and J. Chang, "On two functional equations originating from number theory," Proceedings of the Indian Academy of Science. In press. 


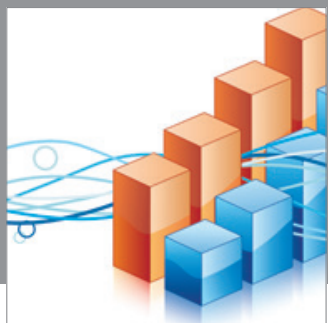

Advances in

Operations Research

mansans

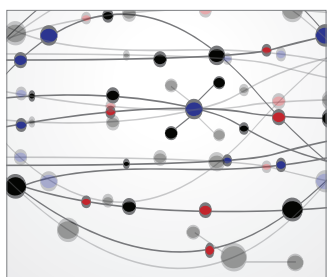

The Scientific World Journal
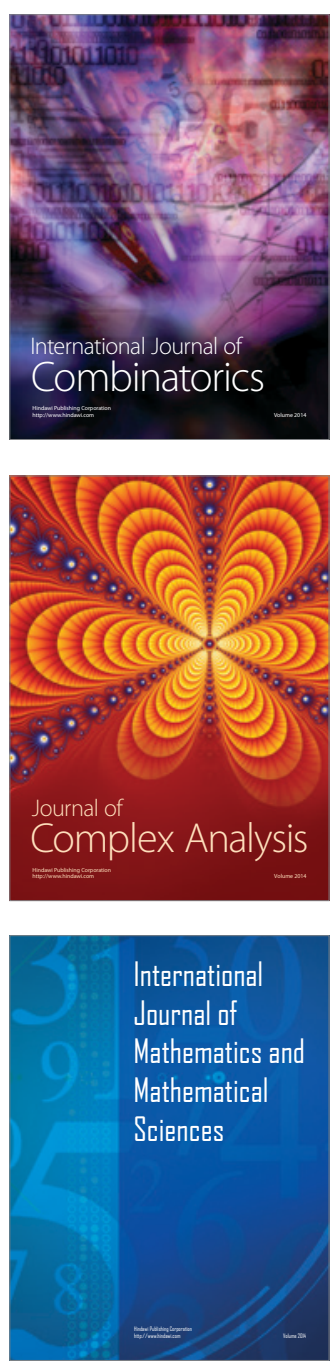
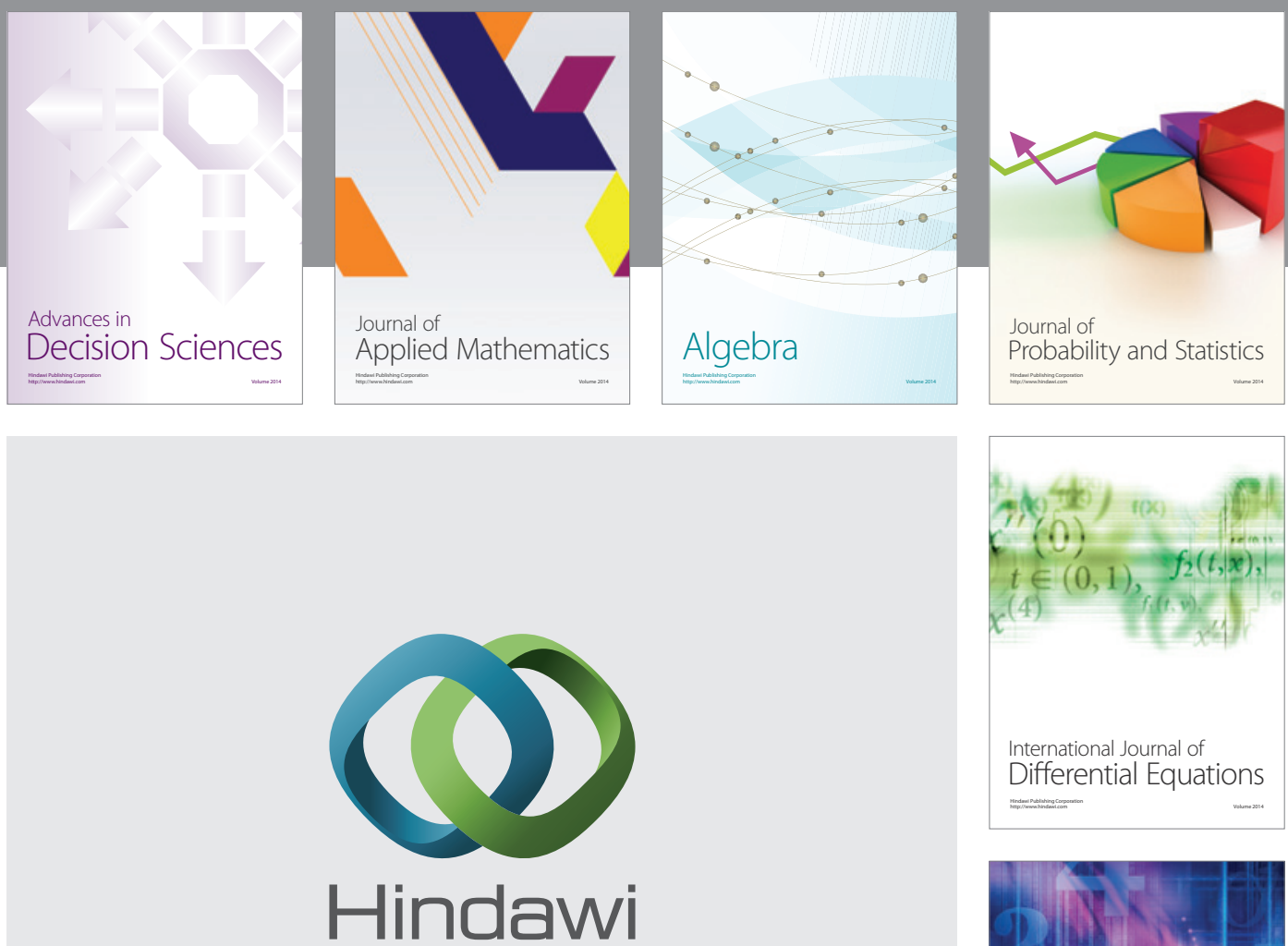

Submit your manuscripts at http://www.hindawi.com
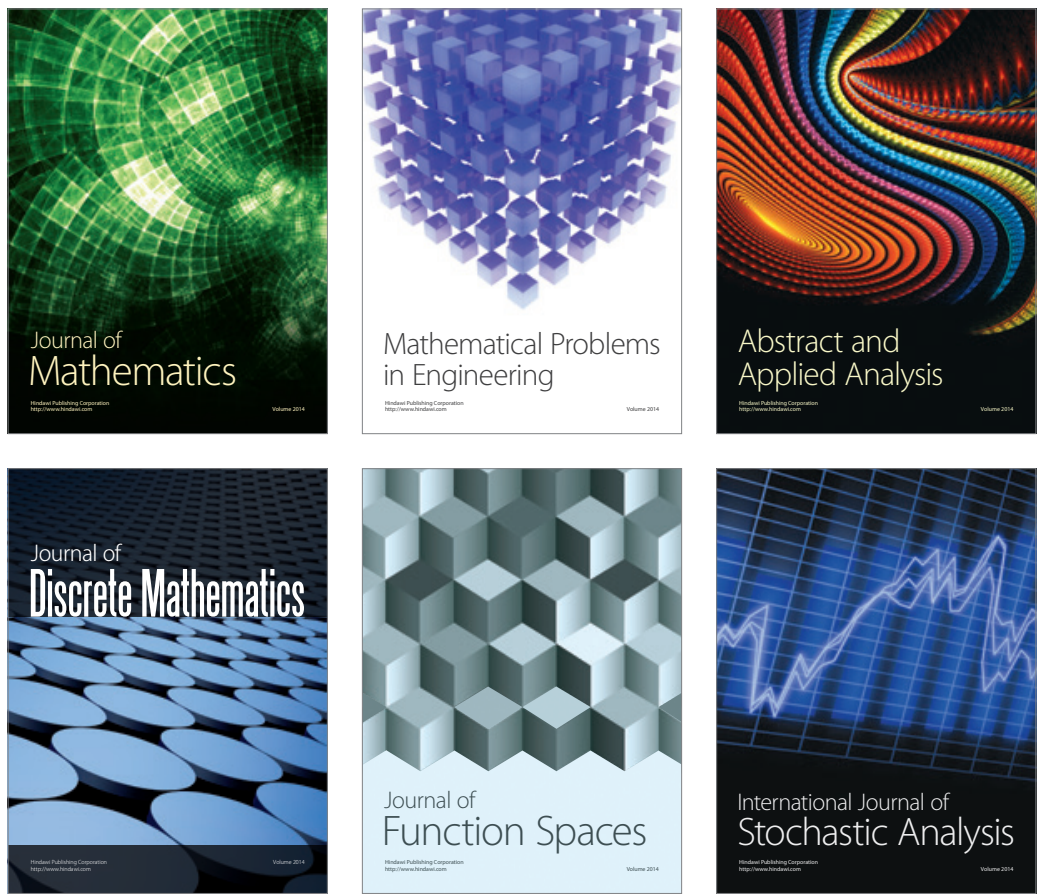

Journal of

Function Spaces

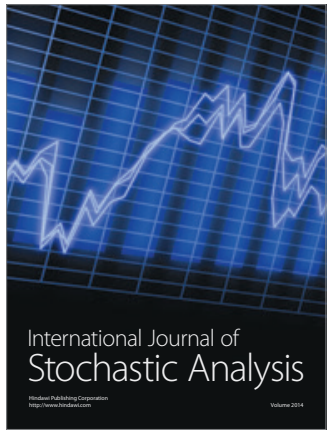

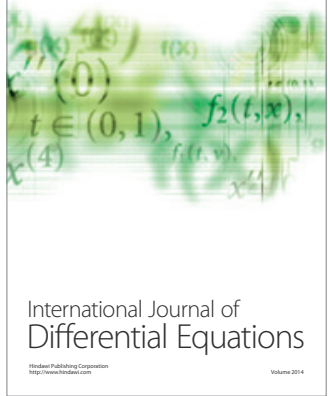
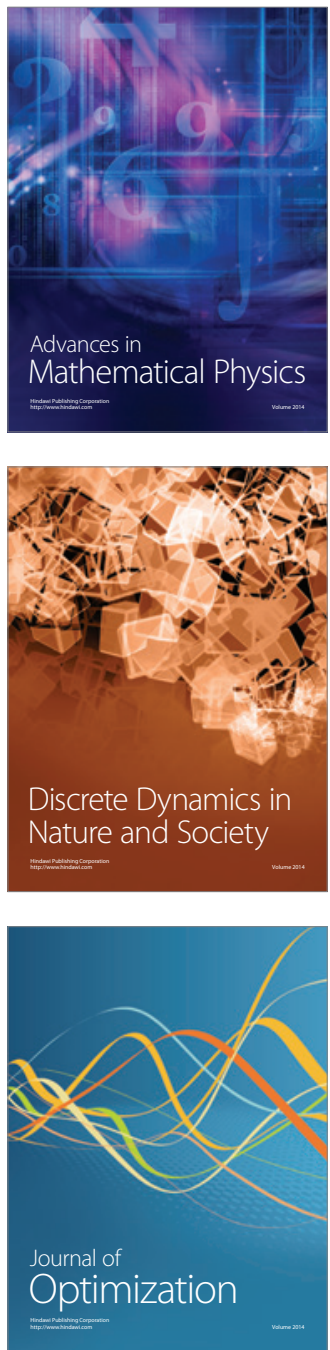